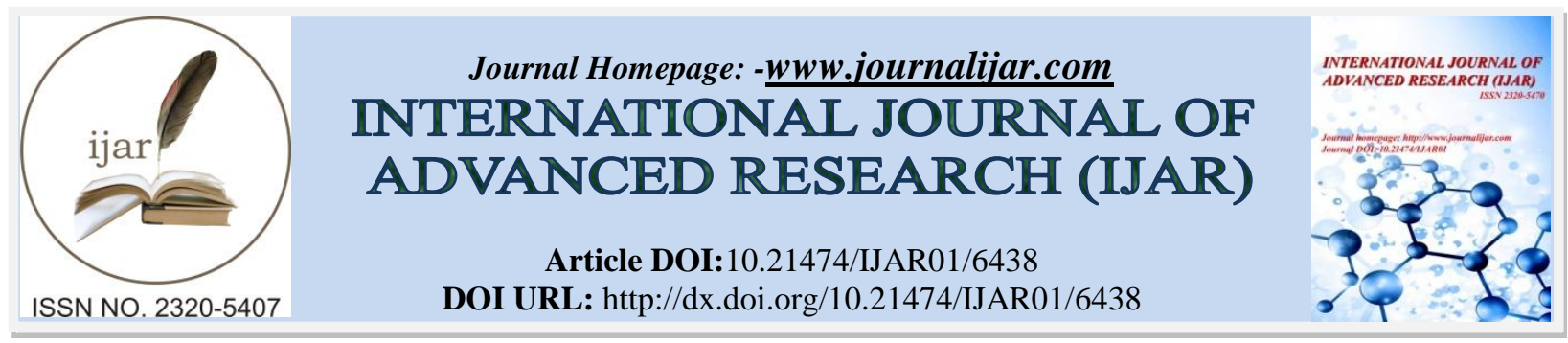

RESEARCH ARTICLE

\title{
CORRELATION BETWEEN CLINICAL FINDINGS AND FUNDUS FLUORESCEIN ANGIOGRAPHY FINDINGS IN PATIENTS WITH PREPROLIFERATIVE DIABETIC RETINOPATHY.
}

\author{
Dr. Isha (Postgraduate Scholar), Dr.Govinder Kumar, Prof. (Dr.) Reyaz Ahmad Untoo (Professor \&Head, \\ Ophthalmology. \\ Principal/Dean, SKIMS Medical College).
}

\section{Manuscript Info}

Manuscript History

Received: 04 December 2017

Final Accepted: 06 January 2018

Published: February 2018

\begin{abstract}
Fluorescein angiography has been extremely valuable for expanding our knowledge to visualize the chorioretinal circulation and in evaluating retinal vascular disorders. The angiogram is used to determine the extent of damage, to develop treatment plan and to monitor the results of treatment. In diabetic retinopathy the angiogram is useful in identifying the extent of ischemia, the location of micro aneurysms, the presence of intraretinalmicrovascular abnormalities (IRMA) that can only be confirmed on angiogram; neovascularization and the extent of macular edema. Fluorescein angiography is an excellent method to display the retinal capillaries in detail to show the pathologic changes because the retinal pigment epithelium provides a good background. FFA is not only useful for diagnosis but also to gauge the progression and management of diabetic retinopathy (DR). FFA is a therapeutic guide to laser photocoagulation treatment for several retinal vascular diseases. In this study, we aimed at highlighting the problems that can occur in early diagnosis and accurate staging of diabetic retinopathy and importance of fluorescein angiography in monitoring diabetic patients. The present study was a hospital based prospective study conducted from November 2015 to April 2017 in the department of Ophthalmology SKIMS Medical College Hospital, Srinagar. Patients older than 12 years of age suffering from Diabetes Mellitus with clinical findings consistent with pre-proliferative diabetic retinopathy were included for the purpose of the study. Patients younger than 12 years of age, patients with hazy media, pregnant women, immunocompromised status patients, patients with hypersensitivity to fluorescein dye or any contraindication to fluorescein dye were excluded from the study. In our study FFA proved to be an important tool to evaluate the lesions of PPDR, which were not detectable on ophthalmoscopy and slit lamp biomicroscopy, such as areas of capillary nonperfusion. IRMA were also best picked up and differentiated from neovascularisation by means of FFA.
\end{abstract}

Copy Right, IJAR, 2018,. All rights reserved. 


\section{Introduction:-}

Diabetes mellitus is defined as a metabolic disorder of multiple etiologies characterized by chronic hyperglycemia with disturbances of carbohydrates, proteins and fat metabolism resulting from defects in insulin secretion, insulin action, or both ${ }^{1}$. Diabetic retinopathy is damage to the retina caused by complications of diabetes mellitus, which can eventually lead to blindness. Diabetic retinopathy (DR) remains the commonest cause of blindness in the working age population of the developed world. Effective treatment is available if the condition is detected early, before visual symptoms occur. The need for a comprehensive DR screening programme has long been recognized and it is now feasible ${ }^{2}$. It is a silent disease and may only be recognized by the patient when the changes in the retina have progressed to a level, that treatment is complicated and nearly impossible ${ }^{3}$. The structure of retinal vessels is a prominent feature that reveals further information on the state of diseases that are reflected in the form of measurable abnormalities in diameter, color, and tortuosity. Thus, reliable methods of vessel detection that preserve various vessel measurements are needed ${ }^{4}$.

Maculopathy is the advanced stage of diabetic retinopathy, where the injuries are located at the area near the macula (the part of the retina that provides clear vision). If this disease is not diagnosed at its early stages, its progress will eventually lead to blindness. Its symptoms are hidden until late stages of the disease ${ }^{5}$.

Diabetic retinopathy is classified according to the presence or absence of abnormal new vessels as (i) Non proliferative (background/preproliferative) retinopathy, (ii) Proliferative retinopathy.

Each has a different prognosis for vision.

Non proliferative diabetic retinopathy (NPDR) (background/pre-proliferative):-

In the international AAO (American Academy of Ophthalmology) classification, NPDR is graded as: (i) Mild, (ii) Moderate, (iii) Severe

In the NSC-UK (national screening committee United Kingdom) classification, NPDR is graded as: (a) Background (level R1), (b) Pre-proliferative (level R2)

In the Scottish diabetic retinopathy grading scheme, NPDR is graded as: (i) Mild background (levelR1), (ii) Moderate background (levelR2), (iii) Severe background (level R3)

\section{Proliferative diabetic retinopathy (PDR):-}

PDR is described according to:

\section{Location i:-}

New vessels on the disc (NVD) or within 1 disc diameter (DD) of the margin of the disc, (ii) New vessels elsewhere in the retina (NVE) (more than 1DD from the disc).

\section{Severity i:-}

Early PDR, (ii) PDR with high risk characteristics, (iii) Florid PDR, (iv) Gliotic PDR.

"Involutionary" PDR is used to describe new vessels which have regressed in response to treatment or (rarely) spontaneously. The different classifications referred to above can be approximately mapped to each other as shown in table below. Retinopathy which affects the macula is separately described as diabetic maculopathy (DM).

The angiogram is used to determine the extent of damage, to develop treatment plan and to monitor the results of treatment. In diabetic retinopathy the angiogram is useful in identifying the extent of ischemia, the location of micro aneurysms, the presence of intraretinalmicrovascular abnormalities (IRMA) that can only be confirmed on angiogram; neovascularization and the extent of macular edema ${ }^{6}$. Fluorescein angiography is an excellent method to display the retinal capillaries in detail to show the pathologic changes because the retinal pigment epithelium provides a good background. Fundus fluorescein angiography (FFA) is not only useful for diagnosis but also to gauge the progression and management of diabetic retinopathy ${ }^{7}$.

Fundus fluorescein angiography is a therapeutic guide to laser photocoagulation treatment for several retinal vascular diseases. FFA images are clinically used in the treatment of macular edema and they show with certainty the different stages of retinopathy and macular leakage in the cases associated with diabetic maculopathy ${ }^{8}$. 
Fluorescein angiography also has a number of potential side effects. The most common complications are transient nausea that occurs in about $2.9 \%$ of patients, as well as vomiting in $1.2 \%$ of patients ${ }^{9}$.There are also various allergic reactions ranging from mild pruritus and urticaria to severe anaphylaxis and death, although the latter are extremely $\operatorname{rare}^{10,11,12}$. There are no risks or adverse reactions that have been associated with pregnancy, but most physicians avoid performing this test in pregnant patients ${ }^{13}$. It is of note that fluorescein dye is not iodine-based, and patients with an iodine allergy can still receive fluorescein dye. The percentage of reactions in patients who had the previous FA without complication was $1.8 \%$, compared with $48.6 \%$ in patients who had a prior reaction to $\mathrm{FA}^{9}$.

The purpose of this study is to establish an algorithm for diagnosis and treatment as close to ideal, ensuring optimum results, with the reduced incidence of immediate or late complications that arise in the development of diabetic retinopathy. This special part of the work indicates the importance of recognizing diabetic retinopathy at an early stage in order to establish the appropriate therapy and to prevent the risk of blindness.

\section{Material and Methods:-}

The present study was conducted in the Department of Ophthalmology, SKIMS Medical College Hospital, Srinagar, which is a tertiary care hospital located in the summer capital of Jammu and Kashmir.

\section{Study Population:-}

All the diabetic patients attending the ophthalmology OPD or admitted in the ophthalmology ward during the study period.

\section{Inclusion criteria:-}

Patients older than 12 years of age suffering from Diabetes Mellitus with clinical findings consistent with preproliferative diabetic retinopathy.

\section{Exclusion criteria:-}

1 Patients younger than 12 years of age.

2 Patients with hazy media.

3 Pregnant women.

4 Immunocompromised status patients.

5 Patients with hypersensitivity to fluorescein dye.

6 Renal insufficiency.

7 Cardiovascular disease.

8 Patients with vascular occlusion diseases of retina.

9 Patients who have already received anti VEGF's.

10 Patients who have undergone laser photocoagulation therapy.

\section{Study Design:-}

Hospital based prospective study.

\section{Study Period:-}

November 2015 to April 2017.

\section{Testing and Examination protocols included:-}

All the patients suffering from Diabetes Mellitus with clinical findings consistent with pre-proliferative diabetic retinopathy were recorded for the purpose of this study. We took into consideration the following characteristics:

1. Visual acuity and pinhole test using Snellen's chart for literate and E chart for illiterate patient.

2. Near vision using $\mathrm{N}$ chart uniocularly and binocularly.

3. Slit lamp biomicroscope for anterior segment examination.

4. Ophthalmoscopy, including stereoscopic examination of the posterior pole.

5. $90 \mathrm{D}$ examination of the fundus.

6. Intraocular pressure measurement.

7. Gonioscopy if necessary.

8. FFA using Carl Zeiss Meditec AG retinophotograph 
We used the Carl Zeiss Meditec AG retinophotograph of the SKIMS Medical College Bemina for every patient. We used $3 \mathrm{ml}$ of $20 \%$ fluorescein from the original sterile container. For pupil dilation, we used drops of $1 \%$ tropicamide and $10 \%$ phenylephrine. As a general protocol, we originally used a drop of each solution in each eye. If after 10 minutes, we did not get proper pupil dilation, we added another set of drops. A third row of drops was needed especially in the patients with very dark eyes, in patients with glaucoma medication or following cataract surgery. All investigations were made in the presence of a physician specialized in Intensive care, equipped with emergency oxygen source, stethoscope, Sphygmomanometer, intubation kit, intravenous needles, fluids, intravenous medication, epinephrine and an antihistamine. Initial ophthalmologic examination of the people with diabetes included: (i) history (type of diabetes, duration of diabetes, glycemic control, current medications, a history of systemic and ocular pathology); (ii) best corrected visual acuity testing, visual disturbances occur early if the lesions are produced in the macula region and the sudden loss of vision may be due to large retinal hemorrhages involving the macula, vitreous bleeding or retinal detachment; (iii) slit lamp biomicroscopy examination of the anterior pole before mydriasis to identify iris neovasculature; (iv) direct and indirect ophthalmoscopy, including stereoscopic examination of the posterior pole; (v) intraocular pressure measurement.

Regarding the complex methods of investigation, we used fluorescein angiography (FA) of patients, who were informed and explained about the importance and necessity of these investigations, after obtaining their written consent.

\section{Fluorescein angiography technique:-}

As with any medical procedure, fluorescein angiography (FA) outcomes are improved when patients are explained the technique, so that they understand what will happen during the test. These explanations have the role to banish any fears and preconceptions, being an integral part of the informed consent necessary before the examination. We prepared the patient for at least 30 minutes before the procedure with mydriatic drops, so that the pupils are dilated sufficiently to allow good quality pictures. Fluorescein injection ( $3 \mathrm{ml}$ of $20 \%)$ was achieved successfully through an angiocath of 21 to 25 gauge needle inserted into the peripheral vein of left arm and fluorescein was injected with $5 \mathrm{ml}$ syringe.

In the presence of diabetic retinopathy, it is recommended a protocol of 7 visual fields. It allows good coverage of the posterior pole and the upper and lower arches.

\section{Results:-}

A total of 76 eyes of 38 patients having PPDR clinically were included for the purpose of study. Mean age of the patients was 52.34 years with a SD of 11.17 years (ranging from 21 to 78years). There were $16(42 \%)$ males and 22 (58\%) females $\left(\chi^{2}=0.95, \mathrm{df}=1, \mathrm{p}=0.330\right)$. Thus the number of males and females was not statistically significant. Family history of diabetes was positive in $15(39 \%)$ patients while $23(61 \%)$ patients had no family history of diabetes. Diabetes mellitus was insulin-dependent in 1 (2.6\%) patients and non-insulin dependent in 37 (97.4\%) patients. The duration of diabetes ranged from 4 to 22 years with a mean duration of $9.63 \pm 4.56$ years. Mean HbA1C level was 6.61 \pm 0.27 . Associated risk factor like hypertension was present in 25 (66\%) patients and13 (34\%) patients had no history of hypertension. 14 patients (37\%) were smokers while 24 patients $(63 \%)$ were non-smokers. Dyslipidemia was present in 8 patients $(21 \%)$ whereas 30 patients $(79 \%)$ had no history of dyslipidemia. Clinically, all three stages of pre-proliferative diabetic retinopathy were seen in the 76 eyes of 38 patients. Mild NPDR was present in 29 eyes, moderate NPDR in 35 eyes and severe NPDR in 12 eyes. On FFA, 6 eyes (8\%) were found to have PDR with one patient having bilateral PDR and 4 patients having unilateral PDR. In the rest of 70 eyes having PPDR, the most common angiographic finding was areas of capillary non-perfusion (CNP areas) seen in 17 eyes (24\%). IRMA and areas of capillary non-perfusion together were seen in 13 eyes (19\%). NVE was seen in 6 eyes proving them to have PDR. Thus among patients with mild NPDR, additional findings were seen in none of the eyes; among those with moderate NPDR, additional findings were seen in 25 out of 35 eyes (72\%) and; among those with severe NPDR, additional findings were seen in 11 out of 12 eyes (92\%). Thus among 76 eyes, a total of 36 eyes $(47 \%)$ showed additional findings on FFA.

Table 1:-Age distribution

\begin{tabular}{|c|c|c|}
\hline Age in Years & No. of Patients & Percentage \\
\hline $20-30$ & 1 & 2.50 \\
\hline $30-40$ & 1 & 2.50 \\
\hline
\end{tabular}




\begin{tabular}{|c|c|c|}
\hline $40-50$ & 18 & 47.0 \\
\hline $50-60$ & 12 & 31.57 \\
\hline $60-70$ & 4 & 10.52 \\
\hline $70-80$ & 2 & 5.00 \\
\hline
\end{tabular}

Table 2:- Gender distribution

\begin{tabular}{|c|c|c|}
\hline Gender & No. of Patients & Percentage \\
\hline Male & 16 & 42.0 \\
\hline Female & 22 & 58.0 \\
\hline Total & 38 & 100.0 \\
\hline \multicolumn{2}{|c|}{ Table 3: Family history of diabetes } \\
\hline Family History & No. of Patients & Percentage \\
\hline Yes & 15 & 39.0 \\
\hline No & 23 & 61.0 \\
\hline Total & 38 & 100.0 \\
\hline
\end{tabular}

Table 4:- Associated Risk Factors

\begin{tabular}{|c|c|c|}
\hline Risk Factors & No. of Patients & Percentage \\
\hline Dyslipidemia & 8 & 21.0 \\
\hline Smoking & 14 & 36.8 \\
\hline Hypertension & 25 & 65.7 \\
\hline
\end{tabular}

Table 5:- Stage of NPDR

\begin{tabular}{|c|c|c|}
\hline \multicolumn{2}{|c|}{} \\
\hline Clinical Stage of NPDR & No. of eyes & Percentage \\
\hline Mild NPDR & 29 & $38 \%$ \\
\hline Moderate NPDR & 35 & $46 \%$ \\
\hline Severe NPDR & 12 & $16 \%$ \\
\hline
\end{tabular}

Table 6:- FFA Findings

\begin{tabular}{|c|c|c|}
\hline \multicolumn{3}{|c|}{} \\
\hline FFA Findings & No. of eyes & Percentage \\
\hline NVE & 6 & $15.7 \%$ \\
\hline IRMA with CNP areas & 13 & $34.2 \%$ \\
\hline CNP areas & 17 & $44 . \%$ \\
\hline
\end{tabular}

Table 7:- Additional FFA findings

\begin{tabular}{|l|l|c|c|}
\hline \multicolumn{2}{|l|}{} \\
\hline \multirow{3}{*}{ Mild NPDR } & Additional finding & No. of eyes & Percentage \\
\hline \multirow{4}{*}{ Moderate NPDR } & CNP areas & 0 & $0 \%$ \\
\cline { 2 - 4 } & IRMA with CNP areas & 0 & $0 \%$ \\
\cline { 2 - 4 } & NVE & 0 & $0 \%$ \\
\cline { 2 - 4 } & No additional finding & 29 & $100 \%$ \\
\cline { 2 - 4 } & CNP areas & 17 & $49 \%$ \\
\cline { 2 - 4 } & IRMA with CNP areas & 8 & $0 \%$ \\
\cline { 2 - 4 } & NVE & 0 & $28 \%$ \\
\cline { 2 - 4 } & No additional finding & 10 & $0 \%$ \\
\hline \multirow{3}{*}{ Severe NPDR } & CNP areas & 0 & $42 \%$ \\
\cline { 2 - 4 } & IRMA with CNP areas & 5 & $50 \%$ \\
\cline { 2 - 4 } & NVE & 6 & $8 \%$ \\
\cline { 2 - 4 } & No additional finding & 1 & $0 \%$ \\
\hline
\end{tabular}




\section{Discussion:-}

Diabetic retinopathy (DR) is a vascular disorder affecting the microvasculature of the retina. It is estimated that diabetes mellitus affects 4 per cent of the world's population, almost half of whom have some degree of DR at any given time ${ }^{14}$. DR occurs both in type 1 and type 2 diabetes mellitus and has been shown that nearly all type 1 and 75 per cent of type 2 diabetes will develop DR after 15 year duration of diabetes as shown in earlier epidemiological studies $^{15,16}$. In the western population, DR has been shown to be the cause of visual impairment in 86 per cent of type 1 diabetic patients and in 33 per cent of type 2 diabetic patients ${ }^{17}$.

In India with the epidemic increase in type 2 diabetes mellitus as reported by the World Health Organization $(\mathrm{WHO})^{18}$, diabetic retinopathy is fast becoming an important cause of visual disability. Visual disability from diabetes is a significant public health problem; however this morbidity is largely preventable and treatable. If managed with timely intervention, the quality of life can be preserved. Diabetic retinopathy is primarily classified into non-proliferative DR (NPDR), formerly termed simple, or background retinopathy, and proliferative DR (PDR).

The prevalence of DR varies in type 1 and type 2 diabetes. In type I diabetes in the EURODIAB IDDM complications study, which included subjects attending 31 European diabetes centres, the prevalence of DR ranged between 25-60 per cent ${ }^{19}$. In India, there is a paucity of data on the prevalence of DR in type 1 diabetes mellitus.

Lack of symptoms and the insidious onset of type 2 diabetes may result in development of DR at an early stage ${ }^{20}$. Often DR is detected when type 2 diabetes is diagnosed. In our study $97.4 \%$ patients were type 2 diabetics.

Studies have shown varying results when predicting gender as a risk factor for developing DR. In the Joslin clinic patients, there appeared to be excess females over males in the older-onset group, however among those with PDR, males were equal to females ${ }^{21}$. In the clinic cohort in Chennai DR appeared to be prevalent more in the males compared to females (sex ratio 2: 1) ${ }^{22}$. A similar preponderance has been reported from the CURES Eye study ${ }^{23}$, UKPDS study ${ }^{24}$ and the Hyderabad study ${ }^{25}$. In our study, there were $58 \%$ females and $42 \%$ males.

The duration of diabetes is probably the strongest predictor for development and progression of retinopathy. In the Wisconsin Epidemiologic Study of Diabetic Retinopathy (WESDR), the widest and most prolonged population based ophthalmologic survey, reported that higher prevalence of DR was associated with longer duration of diabetes $^{26}$. In persons with type 1 diabetes with less than 5 year of duration, the prevalence of retinopathy was about 10 per cent, whereas it ranged from 25 to 40 per cent in individuals with type 2 diabetes.

In India, virtually all studies have shown an increased prevalence of DR as the duration of diabetes increased ${ }^{23,25,27}$. In the study conducted by Dandonaet $a l^{25}$ in type 2 diabetes, it is reported that 87.5 per cent of those with $>15$ years duration of diabetes had DR compared with 18.9 per cent of those who had <15 years duration. In the CURES Eye study $^{23}, 41.8$ per cent had DR after 15 years of diabetes and severity of DR proportionally increased with longer duration of diabetes. In our study the duration of diabetes ranged from 4-22 years, with 12 years in patient with type 1 diabetes.

Increased blood pressure has been hypothesized, through the effects of increased sheer stress of blood flow, to damage the retinal capillary endothelial cells in eyes of people with diabetes ${ }^{28}$. The possible mechanisms by which hypertension may affect DR are haemodynamic (impaired auto regulation and hyperperfusion) and through VEGF (vascular endothelial growth factor). This hypothesis has been supported by observations from clinical studies which showed an association between hypertension and the presence and severity of retinopathy in people with diabetes $^{29,30}$.

The UKPDS showed that the incidence of retinopathy was associated with systolic blood pressure ${ }^{31}$, while in the WESDR, diastolic blood pressure was a significant predictor of progression of diabetic retinopathy to PDR over 14 years of follow up in patients with type 1 diabetes $^{32}$. In the Indian context, hypertension was not a significant confounding factor in the CURES Eye study, however uncontrolled hypertension did influence the progression of $\mathrm{DR}^{23}$. In our study, $66 \%$ patients had a history of hypertension.

Individuals with elevated total serum cholesterol, low-density lipoprotein (LDL) cholesterol or triglyceride levels are more likely to have or develop retinal hard exudates, which can be associated with risk of vision loss, independent of the extent of macular oedema ${ }^{33}$. Several investigators have reported on the association of lipids with 
DR, but the results have not been consistent. The ETDRS ${ }^{34}$ and the WESDR group ${ }^{35}$ found a statistically significant association between elevated serum total cholesterol and LDL cholesterol and the severity of retinal hard exudation in patients with DR. In our study, only $21 \%$ patients had a history of dyslipidemia.

The early detection of diabetic retinopathy leads to a marked reduction of morbidity due to visual loss. Major international studies indicate that therapy is best instituted before serious complications develop.

A study made by Harding et $a l^{36}$ suggested that screening of diabetic retinopathy prevents blindness but because of inadequacies of current screening programs, many diabetic patients never receive treatment before developing severe visual loss. After appropriate screening, early laser photocoagulation prevents severe visual loss. Several alternative screening methods exist like direct ophthalmoscopy, various methods of fundus photography, slit lamp biomicroscopy and FFA ${ }^{36}$.

Until the early 1980s, several reports noted that photography was equivalent to angiography in detecting retinopathy while ophthalmoscopy was not as effective as either of the two methods ${ }^{37}$. Others reported angiography to be more sensitive $^{38}$. In the late 1980s, and after seven-field stereoscopic photography and FFA had been regarded as the gold standard, the ETDRS report no. 5 supported the reliability of both clinical and photographic methods to assess retinopathy ${ }^{39,40}$.

In 1992, the review article by Singer et al listed the sensitivity/specificity of different techniques, showing that FFA was the gold standard ${ }^{41}$. Until the late1990s, the degree of peripheral diabetic retinal changes based on grading of fundus photographs was comparable to that based on angiograms.

In a study by Adhi and Associates ${ }^{37}$ diabetic retinopathy was identified in large number of patients, either focal or scatter laser photocoagulation was done after identifying leaking spots or capillary non-perfusion on fluorescein angiography.

In our study FFA had been used as an important tool to evaluate the lesions of PPDR, which were not detectable on ophthalmoscopy and slit lamp biomicroscopy, such as areas of capillary nonperfusion. IRMA were also best picked up and differentiated from neovascularisation by means of FFA.

By observing the change of fluorescence to detect and quantify areas of leakage and capillary nonperfusion, Philips and coworkers ${ }^{40}$ believed this technique is sufficiently sensitive and robust for clinical use. FFA confirms a presumed diagnosis, determines the course of treatment and documents the finding that may change over time ${ }^{39}$.

In 1993, Sato, Kamata, Matsui ${ }^{40}$ classified 155 eyes (106 patients) affected by PPDR into three sub-groups on the basis of FFA. Mild type with soft exudates and without apparent non-perfused areas on fluorescein angiography (39 eyes), moderate type with demonstrable non-perfused areas on angiography (103 eyes) and severe type with soft exudates, venous beading and non-perfused areas on angiogram (13 eyes). The courses of these three sub-groups were analyzed after one year of follow up. After one year the population developing PDR was 0\% in mild type, $18 \%$ in moderate type and $46 \%$ in severe type.

Sato and Lee ${ }^{41}$ conducted another study in 2002 based on their sub-classification of PPDR proposed earlier ${ }^{40}$. They followed up 54 patients (95 eyes) with PPDR for at least 2 years and found out that proportion developing PDR was $24 \%$ in mild type and $60 \%$ in moderate type. In mild type eyes, the rate of progression to moderate type was $56 \%$ and further progression from moderate to PDR occurred in $43 \%$.

Based on the above results they concluded that their sub-classification of PPDR on FFA could be applied to the early management of the patients with PPDR to prevent vision threatening complications.

In ETDRS ${ }^{42}$, PPDR had $15 \%$ chances and very severe PPDR had $45 \%$ chances of developing PDR within one year. In our study FFA in eyes having PPDR evaluated areas of capillary non-perfusion in 24\%, IRMA and capillary dropout in $19 \%$ of patients.

A retrospective study on reperfusion of occluded capillary bed in diabetic retinopathy done by Takahashi $e t a l^{43}$ reviewed 292 fluorescein angiograms of 94 eyes of 74 patients with diabetic retinopathy. Reperfusion of occluded 
capillary beds was observed in $65(69 \%)$ of 94 eyes. Reperfusion was characterized by re-canalization in 22 (34\%) of 65 eyes or by intraretinal revascularization in $54(83 \%)$ of 65 eyes. In our study intraretinal micro vascular abnormalities were observed in $19 \%$ of patients and areas of capillary non-perfusion in $43 \%$ patients.

In moderate and severe NPDR patients, FFA detected new vessels, and other findings associated with ischemia. FFA showed findings in addition to clinical biomicroscopy findings in $72 \%$ and $92 \%$ eyes with moderate and severe NPDR respectively, in our study.

However, the significance of FFA appears to be questionable in patients with mild NPDR because the detection rate in such cases is quite low. FFA being expensive, time-consuming, and invasive procedure, a suitable compromise would be to perform FFA in all cases of severe NPDR and cases of moderate NPDR with findings suggestive of ischemic changes like cotton wool spots.

\section{Conclusion:-}

In our study FFA proved to be an important tool to evaluate the lesions of PPDR, which were not detectable on ophthalmoscopy and slit lamp biomicroscopy, such as areas of capillary nonperfusion. IRMA were also best picked up and differentiated from neovascularisation by means of FFA. By observing the change of fluorescence to detect and quantify areas of leakage and capillary nonperfusion, this technique is sufficiently sensitive and robust for clinical use. FFA confirms a presumed diagnosis, determines the course of treatment and documents the finding that may change over time. Sub-classification of PPDR on FFA could be applied to the early management of the patients with PPDR to prevent vision threatening complications. The significance of FFA appears to be questionable in patients with mild NPDR because the detection rate in such cases is quite low. FFA being expensive, timeconsuming, and invasive procedure, a suitable compromise would be to perform FFA in all cases of severe NPDR and cases of moderate NPDR with findings suggestive of ischemic changes like cotton wool spots.

\section{Bibliography:-}

1. Definition and diagnosis of diabetes mellitus and intermediate hyperglycaemia. Geneva, World Health Organization, 2006.

2. D. Usher, M. Dumsky, M. Himaga, T. H. Williamson, S. Nussey and J. Boyce. Automated detection of diabetic retinopathy in digital retinal images: a tool for diabetic retinopathy screening. Journal of the British Diabetic Association 2004; Vol. 21, No.1: pp. 84-90.

3. P.N. JebaraniSargunar and R. Sukanesh. Exudates Detection and Classification in Diabetic Retinopathy Images by Texture Segmentation Methods. International Journal of Recent Trends in Engineering 2009 Nov; Vol. 2,No. 4: pp. 148-150.

4. Luo Gang OpasChutatape and Shankar M. Krishnan. Detection and Measurement of Retinal Vessels in Fundus Images Using Amplitude Modified Second-Order Gaussian Filter. IEEE Transactions on Biomedical Engineering 2002 Feb; Vol. 49, No.2: pp. 168-172.

5. MonirehEsnaashari, S. AmirhassanMonadjemi and GholamaliNaderian. A Content-based Retinal Image Retrieval Method for Diabetes Related Eye Diseases Diagnosis. International Journal of Research and Reviews in Computer Science (IJRRCS) 2011 Dec; Vol. 2, No. 6: pp. 1222-1227.

6. Diabetes Care, Diabetic Retinopathy and Diabetic Macular Edema. 2003 Sept; Vol. 26, No. 9: p. 2653 - 2664.

7. Early Treatment Diabetic Retinopathy Study Research Group: ETDRS report No 1: photocoagulation for diabetic macular edema. Arch Ophthalmol 1985; 103: 1796-1806.

8. Swapna T R, ChandanChakroborty. Diabetic maculopathy detection using fundus florescein angiogram imagesa review. International Journal of Research in Engineering and Technology. 2014 Dec; Vol. 03 Special Issue15: IWCPS-2014.

9. Kwiterovich KA, Maguire MG, Murphy RP, Schachat AP, Bressler NM, Bressler SB, et al. Frequency of adverse systemic reactions after fluorescein angiography. Results of a prospective study.Ophthalmology.1991;98:1139-42.

10. The diagnosis and management of anaphylaxis. Joint Task Force on Practice Parameters, American Academy of Allergy, Asthma and Immunology, American College of Allergy, Asthma and Immunology, and the Joint Council of Allergy, Asthma and Immunology. J Allergy ClinImmunol. 1998;101:S465-528.

11. Fineschi V, Monasterolo G, Rosi R, Turillazzi E. Fatal anaphylactic shock during a fluorescein angiography. Forensic Sci Int. 1999;100:137-42. 
12. Hitosugi M, Omura K, Yokoyama T, Kawato H, Motozawa Y, Nagai T, et al. An autopsy case of fatal anaphylactic shock following fluorescein angiography: A case report. Med Sci Law.2004;44:264-5.

13. Berkow JW, Flower RW, Orth DH, Kelley JS. 2nd ed. San Francisco: American Academy of Ophthalmology; 1997. Fluorescein and Indocyanine Green Angiography.

14. Aiello LP, Gardner TW, King GL, Blankenship G, Cavallerano JD, Ferris FL 3rd, et al. Diabetic retinopathy. Diabetes Care 1998; 21: 143-56.

15. Klein R, Klein BE, Moss SE, Davis MD, DeMets DL. The Wisconsin Epidemiologic Study of Diabetic Retinopathy. II. Prevalence and risk of diabetic retinopathy when age at diagnosis is less than 30 years. Arch Ophthalmol1984; 102: 520-6.

16. Rema and Pradeepa. Prevalence and risk factors of diabetic retinopathy 305 Klein R, Klein BEK, Moss SE, Davis MD, DeMets DL. The Wisconsin epidemiologic study of diabetic retinopathy III. Prevalence and risk of diabetic retinopathy when age at diagnosis is 30 or more years. Arch Ophthalmol1984; 102: 527-32.

17. Klein R, Klein BE, Moss SE. Visual impairment in diabetes. Ophthalmology 1984; 91: 1-9.

18. Wild S, Roglic G, Green A, Sicree R, King H. Global prevalence of diabetes, estimates for the year 2000 and projections for 2030. Diabetes Care 2004; 27: 1047-53.

19. Abrahamian H, Hornlein B, Gurdet C, Willinger C, Zaruba E, Irsigler K. Insulin-dependent diabetes mellitus: "EURODIAB IDDM Complications Study" - results from the Vienna center. Wien KlinWochenschr 1994; 106:136-140.

20. Harris MI. Undiagnosed NIDDM: clinical and public health issues. Diabetes Care 1993; 16: 642-52.

21. Aiello LM, Rand LI, Briones JC, Wafai MZ, Sebestyen JG. Diabetic retinopathy in Joslin Clinic patients with adult onset diabetes. Ophthalmology 1981; 88: 619-23.

22. Rema M, Ponnaiya M, Mohan V. Prevalence of retinopathy in non-insulin dependent diabetes mellitus at a diabetes centre in southern India. Diabetes Res Clin Pract1996; 34: 29-36.

23. Rema M, Premkumar S, Anitha B, Deepa R, Pradeepa R, Mohan V. Prevalence of diabetic retinopathy in urban India: The Chennai Urban Rural Epidemiology Study (CURES) Eye Study, I. Invest Ophthalmol Vis Sci2005; 46: 2328-33.

24. Harris MI, Klein R, Welborn TA, Knuiman MW. Onset ofNIDDM occurs at least 4-7 years before clinical diagnosis. Diabetes Care 1992; 15: 815-9.

25. Dandona L, Dandona R, Naduvilath TJ, McCarty CA, RaoGN. Population based assessment of diabetic retinopathy in an urban population in southern India. Br J Ophthal1999; 83: 937-40.

26. Klein R, Davis MD, Moss SE, Klein BE, DeMets DL. The Wisconsin Epidemiologic Study of Diabetic Retinopathy.A comparison of retinopathy in younger and older onset diabetic persons. AdvExp Med Biol1985; 189: 321-35.

27. Rema M, Shanthirani CS, Deepa R, Mohan V. Prevalence of diabetic retinopathy in a selected South Indian Population - The Chennai Urban Population Study (CUPS).Diabetes Res Clin Pract2000; 50: S252.

28. Kohner EM. Diabetic retinopathy. Br Med Bull 1989; 45:148-73.

29. Rema M, Sujatha P, Pradeepa R. Visual outcomes of panretinal photocoagulation in diabetic retinopathy at oneyear follow-up and associated risk factors. Indian J Ophthalmol2005; 53: 93-9.

30. Fujisawa T, Ikegami H, Yamato E, Kawaguhi Y, Ueda H, Shintani M, et al. Association of plasma fibrinogen level and blood pressure with diabetic retinopathy and renal complications associated with proliferative diabetic retinopathy in type II diabetes mellitus. Diabet Med 1999;16: 522-6.

31. Kostraba JN, Klein R, Dorman JS, Becker DJ, Drash AL, Maser RE, et al. The Epidemiology of Diabetes Complications Study. IV. Correlates of diabetic background and proliferative retinopathy. Am J Epidemiol1991; 133:381-91.

32. Klein R, Klein BE, Moss SE, Cruickshanks KJ. The Wisconsin Epidemiologic Study of Diabetic Retinopathy: XVII. The 14-year incidence and progression of diabetic retinopathy and associated risk factors in Type 1 diabetes. Ophthalmology 1998; 105:1801-15.

33. Chew EY, Klein ML, Ferris FL III, Remaley NA, MurphyRF, Chantry K, et al. Association of elevated serum lipid levels with retinal hard exudates in diabetic retinopathy.Early Treatment Diabetic Retinopathy Study (ETDRS) report 22. Arch Ophthalmol1996; 114: 1079-84.

34. Ferris FL 3rd, Chew KY, Hoogwerf BJ. Serum lipids and diabetic retinopathy. Early Treatment Diabetic Retinopathy Study Research Group. Diabetes Care 1996; 19: 1291-3.

35. Klein BEK, Moss SE, Klein R, Surawicz TS. The Wisconsin Epidemiologic Study of Diabetic Retinopathy, XIII: relationship between serum cholesterol to retinopathy and hard exudate. Ophthalmology 1991; 98: 1261-5. 
36. Harding SP, Broadbent DM, Neoh C, et al. Sensitivity and specificity of photography and direct ophthalmoscopy in screening for sight threatening eye disease: The Liverpool Diabetic Eye Study, BMJ 1995; 311: 1131-5.

37. Adhi MI, Ansari, AA, Aziz MU, et al. Clinical Audit of fundus fluorescein angiograms, Pak J Ophthalmol. 1997; 13: 3-7.

38. Phillips RP, Rose PG, Sharp PF, et al. Use of temporal information to quantify vascular leakage in fluorescein angiography of retina, ClinPhysphysiol Meas. 1990; 11: 81-5.

39. Muhammad S. Fundus fluorescein angiography, J Postgrad Med InstPesh. 1998; 12: 8-16.

40. Sato Y, Kamata A, Matsui M. Sub-classification of pre-proliferative diabetic retinopathy, Jpn J Ophthalmol. 1993; 37: 490-8.

41. Sato Y, Lee Z., The sub-classification and long-term prognosis of pre-proliferative diabetic retinopathy, Jpn J Ophthalmol. 2002; 46: 323-9.

42. Early treatment diabetic retinopathy study research group.Early Photocoagulation for diabetic retinopathy. ETDRS report 9, Ophthalmology. 1991; 98: 766-85.

43. Takahashi K, Kishi S, Muraoka K et al. Reperfusion of occluded capillary beds in diabetic retinopathy. Am J Ophthalmol. 1998; 126: 791-7. 